\title{
Análise da mortalidade por COVID-19 e obesidade como fator de risco
}

\author{
Analysis of mortality from COVID-19 and obesity as a risk factor \\ Análisis de la mortalidad por COVID-19 y la obesidad como factor de riesgo
}

\section{Resumo}

A obesidade é causada pelo aumento da massa de tecido adiposo, sendo considerada uma doença multifatorial que pode ser evitada. O principal fator desencadeante é o desequilíbrio energético, devido ao alto nível de calorias consumidas e baixa atividade energética. No Brasil, a obesidade cresceu $67,8 \%$, sendo um dos fatores mais expressivo em pacientes com COVID-19. A COVID-19 (coronavirus disease 2019) é uma infecção respiratória causada pelo coronavírus SARSCoV-2, que pode evoluir de um quadro gripal para Síndrome Respiratória Aguda Grave até a morte. A pandemia atual apresentou um início recente e um ritmo rápido no seu desenvolvimento, devido sua elevada taxa de transmissão e distribuição global. O conjunto de evidências científicas ainda é escasso, cada surto que surge é uma oportunidade de se obter mais informações sobre o vírus. Esse trabalho tem por objetivo contribuir para ampliar o conhecimento da pandemia da COVID-19 avaliando a taxa de mortalidade por COVID-19 associada à obesidade como fator de risco, observando os boletins epidemiológicos de abril de 2020 a agosto de 2021 da Doença pelo Coronavírus 2019, tendo como destaque os óbitos, segundo idade, sexo e região de maior prevalência. Assim, como resultado dos dados coletados, observa-se que a região Centro-Oeste é a de maior incidência, o sexo masculino mais prevalente, e a obesidade é o principal fator de risco para menores de 60 anos. Portanto, faz-se necessário que o país intensifique ações de vigilância de fatores agravantes, como a obesidade, sendo um fator de risco modificável.

Palavras-chave: Pandemia; COVID-19; SARS-CoV-2; Coronavírus; Comorbidade.

\begin{abstract}
Obesity is caused by an increase in the mass of adipose tissue, being considered a multifactorial disease that can be avoided. The main triggering factor is energy imbalance, due to the high level of calories consumed and low energy activity. In Brazil, obesity grew by $67.8 \%$, being one of the most significant factors in patients with COVID-19. COVID19 (coronavirus disease 2019) is a respiratory infection caused by the SARS-CoV-2 coronavirus, which can progress from a flu to Severe Acute Respiratory Syndrome until death. The current pandemic has a recent onset and a rapid pace of development due to its high rate of transmission and global distribution. The body of scientific evidence is still sparse, each outbreak that arises is an opportunity to obtain more information about the virus. This work aims to contribute to increasing knowledge of the COVID-19 pandemic by evaluating the mortality rate from COVID-19 associated with obesity as a risk factor, observing the epidemiological bulletins from April 2020 to August 2021 of Coronavirus Disease 2019, with emphasis on deaths, according to age, sex and region of greatest prevalence. Thus, as a result of the collected data, it is observed that the Midwest region has the highest incidence, the male gender is more prevalent and obesity is the main risk factor for children under 60 years of age. Therefore, it is necessary for the country to intensify surveillance actions for aggravating factors, such as obesity, which is a modifiable risk factor.
\end{abstract}

Keywords: Pandemic; COVID-19; SARS-CoV-2; Coronavirus; Comorbidity. 


\begin{abstract}
Resumen
La obesidad es causada por un aumento de la masa de tejido adiposo, siendo considerada una enfermedad multifactorial que puede evitarse. El principal factor desencadenante es el desequilibrio energético, debido al alto nivel de calorías consumidas y la baja actividad energética. En Brasil, la obesidad creció en un 67,8\%, siendo uno de los factores más significativos en los pacientes con COVID-19. COVID-19 (enfermedad por coronavirus 2019) es una infección respiratoria causada por el coronavirus SARS-CoV-2, que puede progresar desde una gripe hasta un síndrome respiratorio agudo severo hasta la muerte. La pandemia actual tiene un inicio reciente y un rápido ritmo de desarrollo debido a su alta tasa de transmisión y distribución global. El cuerpo de evidencia científica aún es escaso, cada brote que surge es una oportunidad para obtener más información sobre el virus. Este trabajo tiene como objetivo contribuir a incrementar el conocimiento de la pandemia COVID-19 mediante la evaluación de la tasa de mortalidad por COVID19 asociada a la obesidad como factor de riesgo, observando los boletines epidemiológicos de abril de 2020 a agosto de 2021 de la Enfermedad del Coronavirus 2019, con énfasis en las defunciones, según edad, sexo y región de mayor prevalencia. Así, como resultado de los datos recolectados, se observa que la región Medio Oeste tiene la mayor incidencia, el género masculino es más prevalente y la obesidad es el principal factor de riesgo para los menores de 60 años. Por tanto, es necesario que el país intensifique las acciones de vigilancia de factores agravantes, como la obesidad, que es un factor de riesgo modificable.
\end{abstract}

Palabras clave: Pandemia; COVID-19; SARS-CoV-2; Coronavirus; Comorbilidad.

\title{
1. Introdução
}

A obesidade é uma doença multifatorial, que pode ser evitada e tem como seu principal fator o desequilíbrio energético, devido ao alto nível de calorias consumidas e baixa atividade energética (World Health Organization [WHO], 2020). Sua prevalência nas últimas décadas coexiste devido a influência do estilo de vida, sobretudo devido à alimentação inadequada e ao sedentarismo (Ferreira, Szwarcwald, \& Damacena, 2019). Para determinar o acúmulo anormal ou excessivo de gordura é avaliado o índice de massa corporal (IMC), obtido pela divisão do peso, medido em quilogramas, pela altura ao quadrado, medida em metros $\left(\mathrm{kg} / \mathrm{m}^{2}\right)$ (Halpern et al., 2021). Valores maiores ou iguais a $25 \mathrm{~kg} / \mathrm{m}^{2}$ indicam excesso de peso e valores maiores ou iguais a $30,0 \mathrm{~kg} / \mathrm{m}^{2}$ caracterizam obesidade, ainda subdividida em graus (WHO, 2020)

Em 2016, cerca de $13 \%$ da população adulta mundial era obesa, sendo $11 \%$ homens e $15 \%$ mulheres (Associação Brasileira para o Estudo da Obesidade e da Síndrome Metabólica [ABESO], 2021). No Brasil, a obesidade cresceu 67,8\% nos últimos treze anos, saindo de 11,8\% em 2006 para 19,8\% em 2018, de acordo com a Pesquisa de Vigilância de Fatores de Risco e Proteção para Doenças Crônicas por Inquérito Telefônico (Sistema de Vigilância de Fatores de Risco e Proteção para Doenças Crônicas por Inquérito Telefônico [VIGITEL], 2016).

Para ressaltar a importância dessa doença crônica, inflamatória, causada pelo aumento da massa de tecido adiposo, é necessário citar as comorbidades associadas. A obesidade é um fator precursor de doenças crônicas não transmissíveis como a hipertensão arterial sistêmica e diabetes mellitus tipo 2, alguns tipos de câncer, doenças cardiovasculares, distúrbios musculares (Chait \& Hartigh, 2020). O tecido adiposo apresenta funções tais como, estoque energético, isolante térmico, proteção contrachoque mecânicos. É considerado um órgão endócrino que promove o metabolismo de hormônios como adipocinas e citocinas pró-inflamatórias como IL-6 e TNF-alfa e redução da resposta imune inata e adquirida (Fonseca-Alaniz, Takada, Alonso-Vale \& Lima, 2006). No entanto, torna-se disfuncional, promovendo um ambiente pró-inflamatório, hiperlipidêmico e resistente à insulina (Bolsoni-Lopes, Furieri \& Alonso-Vale, 2021).

Tal contexto denota que o mecanismo patológico causado pela obesidade contribui com maior risco de infecções e complicações, devido ao acometimento metabólico e, pela mesma razão, o período de transmissibilidade de infecções virais é maior em relação à população não obesa (Malavazos, Romanelli, Bandera \& Iacobellis, 2020). O acúmulo de tecido adiposo promove um ambiente favorável para o desenvolvimento de maior carga viral e demanda maior tempo para resolução de processos patológicos. Isso porque apresenta menor ação de fagocitose por macrófagos, retardo na apresentação e reconhecimento de antígenos no desenvolvimento de anticorpos, menor ação de leucócitos B e T e diminuição na produção de interferons (Bolsoni-Lopes, Furieri \& Alonso-Vale, 2021). 
Nesse sentido, é relevante destacar que pessoas com doenças crônicas como a obesidade apresentam mais complicações decorrentes da infecção por COVID-19, por conta da presença de características fisiopatológicas como a diminuição de reserva expiratória, a capacidade funcional, a complacência do sistema respiratório e a excursão diafragmática, tendo essas evidências contribuindo para analisar a obesidade como um fator de risco para mortalidade por COVID-19 (Cândido, Moreira, Alexandre, Parente \& Cavalcante, 2021). A obesidade é um fator mais expressivo em pacientes com COVID-19 menores 50 anos, em comparação com os doentes com mais idade. Dessa maneira, indica uma priorização desse grupo para serem vacinados junto com profissionais de saúde, pessoas acima de 60 anos, entre outras comorbidades (Souza \& Buss, 2021). Ademais, a distribuição equitativa em escala global e a organização de toda logística e execução da campanha é de extrema relevância para o acesso igualitário das vacinas (Gregório, Santos \& Graça, 2021).

Sendo assim, observa-se a importância de conhecer e compreender os fatores epidemiológicos para o controle da disseminação da COVID-19. Tendo a obesidade como fator de risco para o agravamento dos quadros de COVID-19 e as medidas de restrição de transmissão do coronavírus que poderão ter consequências para a saúde, a médio e longo prazo, principalmente em relação ao aumento do número de pessoas obesas no Brasil. É importante ressaltar que as resolutividades das ações atuais vão refletir nas consequências e futuro da pandemia. Tendo como agentes dessas ações a tríade governo-sociedade-setor privado, sob as perspectivas social, econômica e, principalmente, da saúde (Malta, et al., 2020). Portanto, o presente estudo teve por objetivo analisar a obesidade como fator de risco para mortalidade por COVID-19, após análise da incidência no Brasil. A partir dos dados coletados pretende-se contribuir para ampliar o conhecimento sobre a pandemia da COVID-19, e ressaltar a importância do planejamento de políticas públicas contra a obesidade, que é um fator de risco modificável.

\section{Metodologia}

Para alcançar os objetivos propostos pelo trabalho, foi feito um estudo observacional, descritivo, subsidiado por evidências científicas, desenvolvida através de pesquisas organizadas em fontes de dados. Promovendo uma construção críticoreflexiva sobre a temática da análise da mortalidade por COVID-19 a obesidade como fator de risco nos anos de 2020 e 2021. Seguindo essa lógica, foram analisados boletins epidemiológicos, que são publicações de caráter técnico-científico, de acesso público, no formato eletrônico, com periodicidade definida para cada doença, com intuito de monitoramento e investigação de cada região. Relacionado ao percentual de obesidade, os dados estão disponíveis no Sistema de Vigilância de Fatores de Risco e Proteção para Doenças Crônicas por Inquérito Telefônico (VIGITEL). Esse sistema tem por base entrevistas de residentes em domicílios com telefone fixo, maiores de 18 anos, em cada uma das capitais dos 26 estados brasileiros e no Distrito Federal. Foram realizadas amostra probabilística com aproximadamente 52 mil entrevistas em 2019. O percentual da obesidade, quando $\mathrm{IMC} \geq 30 \mathrm{~kg} / \mathrm{m}^{2}$, foi avaliado pelo número de indivíduos com obesidade/número de indivíduos entrevistados. Assim, foi calculada a média de cada região brasileira tendo como base a porcentagem de cada estado brasileiro (Brasil - Ministério da Saúde (2021a).

Para avaliar a taxa de óbitos por Síndrome Respiratória Aguda Grave (SRAG) ocasionadas por COVID-19 foram analisados os seguintes boletins epidemiológicos: Semana Epidemiológica (SE) 6 do dia 26 de fevereiro de 2020, quando houve o primeiro caso no Brasil de COVID-19 até 03 de abril de 2020, e SE 34 do primeiro caso até o dia 28 de agosto de 2021 (Brasil - Ministério da Saúde, 2020 a, b). Para avaliar a obesidade como perfil epidemiológico dos óbitos por COVID-19, foram usadas as seguintes variáveis: regiões do Brasil (Norte, Nordeste, Centro-Oeste, Sul e Sudeste), sexo (feminino e masculino) e faixa etária (18 a 59 anos e acima de 60 anos), e realizada a discussão dos dados obtidos. 


\section{Resultados e Discussão}

A pandemia causada pelo novo coronavírus 2019, COVID-19 (coronavirus disease 2019) foi reconhecida pela Organização Mundial da Saúde (OMS) no dia 11 de março de 2020. A infecção por SARS-CoV-2 se dá de pessoa para pessoa, por meio de gotículas e superfícies contaminadas, atinge as vias aéreas superiores e inferiores, iniciando um quadro gripal que pode evoluir para Síndrome Respiratória Aguda Grave, podendo ser necessário sua hospitalização (Bolsoni-Lopes, Furieri \& Alonso-Vale, 2021).

De acordo com a Semana Epidemiológica (SE) 8 de 2021, do Ministério da Saúde, a primeira notificação de um caso confirmado de COVID-19 no Brasil ocorreu no dia 26 de fevereiro de 2020. Desde a primeira notificação até dia 27 de fevereiro de 2021 foram confirmados 10.517.232 casos e 254.221 óbitos por COVID-19, sendo distribuídos de forma diferente entre os estados do Brasil (Brasil - Ministério da Saúde, 2021a). Na literatura já disponível, a letalidade por SARS-CoV-2 foi associada principalmente aos pacientes idosos, imunossuprimidos e presença de comorbidades. Contudo, o decorrer da pandemia vem mostrando uma evolução no registro de casos e mudanças no seu percurso. O conjunto de evidências científicas ainda é insuficiente, cada surto é uma oportunidade de se obter mais informações sobre o vírus (Lana et al., 2020).

A formulação de estratégias para o controle epidemiológico da COVID-19 foi complexa devido à diversidade na clínica e achados de imagem e laboratoriais devido a gravidade. Na admissão de 1.099 pacientes chineses com COVID-19, de 11 de dezembro de 2019 até 29 de janeiro de 2020, foram observados os seguintes principais sintomas: febre (88,7\%), tosse $(67,8 \%)$; náuseas ou vômitos $(5,0 \%)$ e diarreia $(3,8 \%)$. Os achados de tomografia computadorizada de tórax observaram opacidade em vidro fosco $(56,4 \%)$ e sombras irregulares bilaterais $(51,8 \%)$. Além dos exames laboratoriais alterados apresentando linfocitopenia $(83,2 \%)$, trombocitopenia $(36,2 \%)$ e leucopenia $(33,7 \%)$ e elevados níveis de proteína C reativa (Guan et al., 2020).

Ao avaliar a velocidade de propagação do vírus, de acordo com a sua reprodução, que é indicado pelo número médio de casos secundários gerados por caso primário. Para o SARS-CoV-2 sua velocidade varia de 1,6 a 4,1. Como método de comparação a avaliação do H1N1 em 2009 apresentou uma variação de velocidade de propagação de 1,3 e 1,8. A maior notificação no Brasil ocorreu no estado do Paraná com 643 casos por 100 mil habitantes (Lana et al., 2020).

Devido sua rápida transmissibilidade e contaminação, com ausência de intervenções médicas preventivas ou terapêuticas específicas para infecção por SARS-CoV-2 no início da pandemia, foi adotado o uso de intervenções não farmacológicas, como lavagem de mãos, uso de máscara, restrição da circulação social. A restrição social é a medida mais efetiva para evitar a disseminação da doença e achatar a curva de transmissão do coronavírus, no entanto, observa-se mudanças nos estilos de vida e na saúde mental dos cidadãos (Malta, et al., 2020). Junto a isso houve uma redução dos níveis de atividade física, aumento do comportamento sedentário, aumento no consumo de álcool, tabaco e aumento do consumo de alimentos ultraprocessados densos em energia, ricos em gordura e açúcares (por conta do enfraquecimento no abastecimento de alimentos ou acesso limitado a alimentos frescos), durante o período de quarentena no Brasil (Malta et al., 2020).

O rápido avanço no desenvolvimento de vacinas contra a COVID-19 representa uma grande esperança na saúde pública. A Campanha Nacional de Vacinação no Brasil foi oficialmente iniciada em 18 de janeiro de 2021, mas persiste em uma longa caminhada até alcançar a imunidade coletiva equitativa de superar a pandemia (Souza \& Buss, 2021). No entanto, ainda não se sabe se as vacinas irão induzir memória imunológica de longa duração, se haverá necessidade ou não de revacinação anual para novas cepas (Domingues, 2021).

Em relação aos fatores de risco a obesidade é considerada um fator para agravamento da COVID-19, por contribuir para complicações da infecção pelo SARS-CoV2, devido à amplificação do estado de inflamação, danos ao sistema respiratório, cardiovascular e metabolismo da glicose, favorecimento da formação de trombos e a desregulação do sistema imune (Bolsoni- 
Lopes, Furieri \& Alonso-Vale, 2021). Ao analisar a frequência de adultos obesos em todo o território brasileiro a região CentroOeste foi a que apresentou maior média de obesos contaminados em relação as demais regiões do Brasil, como descrito na Tabela 1. Foram observadas as seguintes porcentagens para as capitais da região Centro-Oeste: Campo Grande (MS) 22,5\%, Cuiabá (MT) 22,5\%, Brasília (DF) 19,6\% e Goiânia (GO) 19,5\%, (VIGITEL, 2019).

Tabela 1- Percentual de adultos ( $\geq 18$ anos) com obesidade (IMC $\geq 30 \mathrm{~kg} / \mathrm{m}^{2}$ ), de acordo com as regiões brasileiras.

\begin{tabular}{ll}
\hline Regiões do Brasil & $\%$ \\
\hline Região Centro-Oeste & 21,02 \\
Região Norte & 20,81 \\
Região Nordeste & 19,77 \\
Região Sudeste & 19,77 \\
Região Sul & 19,6 \\
\hline
\end{tabular}

Fonte: Elaborada pelos autores com base nos dados do VIGITEL (2019).

Em relação a obesidade segundo a idade, a frequência foi maior em adultos com 55 a 64 anos (42,3\%), seguido por 45 a 54 anos (24,5\%). A obesidade também foi analisada de acordo com o sexo, sendo semelhante entre homens e mulheres (VIGITEL, 2019). No entanto, ao comparar as duas variáveis (sexo e idade), como descrito na Tabela 2, foi possível observar que a obesidade foi mais frequente em homens até os 64 anos, e mulheres até os 54 anos.

Tabela 2- Percentual de adultos ( $\geq 18$ anos) com obesidade (IMC $\geq 30 \mathrm{~kg} / \mathrm{m}^{2}$ ), das capitais dos estados brasileiros e do Distrito Federal, por sexo e segundo idade (anos).

\begin{tabular}{llll}
\hline & Idade (anos) & \multicolumn{2}{c}{ Sexo } \\
& Total (\%) & Masculino (\%) & Feminino (\%) \\
\hline 18 a 24 & 8,7 & 5,7 & 10,3 \\
25 a 34 & 19,3 & 16,6 & 19,2 \\
35 a 44 & 22,8 & 20,9 & 21,9 \\
45 a 54 & 24,5 & 20,8 & 25,2 \\
55 a 64 & 42,3 & 21,8 & 24,0 \\
65 e mais & 20,9 & 15,8 & 22,7 \\
\hline
\end{tabular}

Fonte: Elaborada pelos autores com base nos dados do VIGITEL, (2019).

Foram analisadas as taxas de mortalidade por COVID-19 associada a obesidade como fator de risco, observando os boletins epidemiológicos de março de 2020 a agosto 2021, de mortes causadas por COVID-19 segundo idade, sexo e região de maior prevalência. As análises indicaram a incidência de obesidade $(20,3 \%)$ e excesso de peso $(55,4 \%)$. Observa-se que a obesidade e suas comorbidades associadas (diabetes, hipertensão arterial), estão relacionadas com o número de óbitos pela COVID-19 (boletim epidemiológico 27).

De acordo com o Boletim Epidemiológico 03, de abril de 2020, o primeiro óbito confirmado no Brasil ocorreu no dia 17 de março de 2020. Até o dia 03 de abril de 2020 foram 359 óbitos, sendo o maior número registrado na região Sudeste com 276 óbitos (4,9\%). A idade com maior número de registros (242 óbitos) foi de 60 anos ou mais (85\%). O sexo masculino com 165 óbitos foi o mais acometido (57,7\%). Dentre esses óbitos $82 \%$ apresentavam pelo menos um fator de risco, sendo os 
principais observados cardiopatia e diabetes, em sua maioria em indivíduos com 60 anos ou mais, conforme apresentado na Figura 1.

Figura 1- Óbitos por COVID-19 no Brasil classificados por grupos de risco e faixa etária.

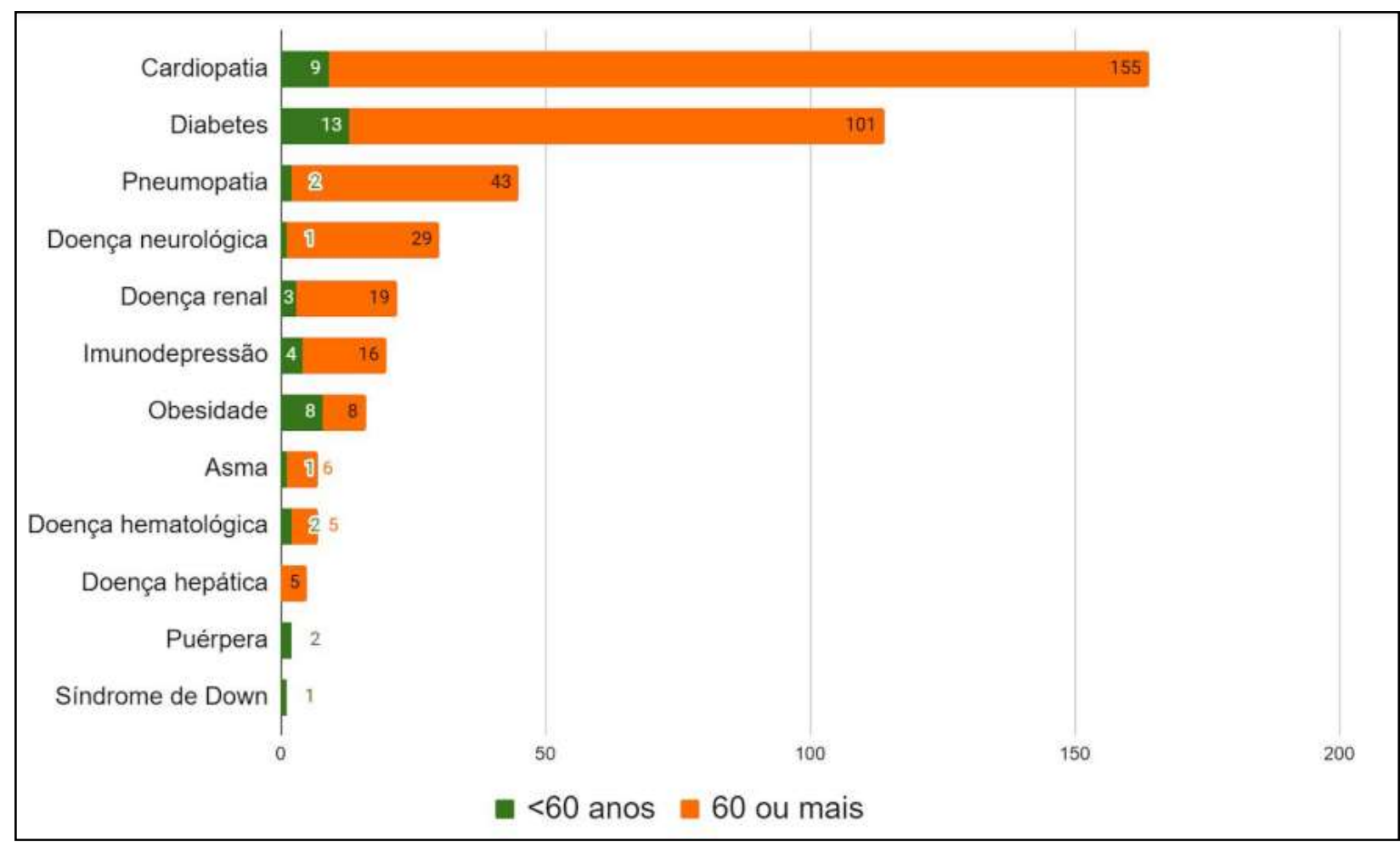

Fonte: Sistema de Informação de Vigilância da Gripe (SIVEP-Gripe, 2021). Dados atualizados em 03 de abril de 2020.

Ao avaliar a taxa de óbitos de SRAG causadas por COVID-19 notificados do primeiro óbito até dia 28 de agosto de 2021 (SE 34), foram confirmados 579.010 óbitos e 20.728 .605 casos por COVID-19 no Brasil. A maior taxa de mortalidade ocorreu na região Centro-Oeste (3,6 óbitos/100 mil habitantes) Entre os 335.151 óbitos de SRAG por COVID-19 notificados em 2021 até a SE 34, 199.234 apresentavam pelo menos uma comorbidade (59,4\%), sendo as principais relatadas diabetes e a obesidade. A maioria com 60 anos ou mais de idade, no entanto, o óbito com obesidade foi maior dentre os menores de 60 anos (24.972 óbitos), como observado na Figura 2. Em relação ao sexo, o mais acometido foi o masculino com 186.004 óbitos por SRAG (Sistema de Informação de Vigilância da Gripe [SIVEP-Gripe], 2021). 
Figura 2- Comorbidades e fatores de risco dos óbitos por Síndrome Respiratória Aguda Grave causadas por COVID-19. Brasil, 2021 até Semana Epidemiológica 34.

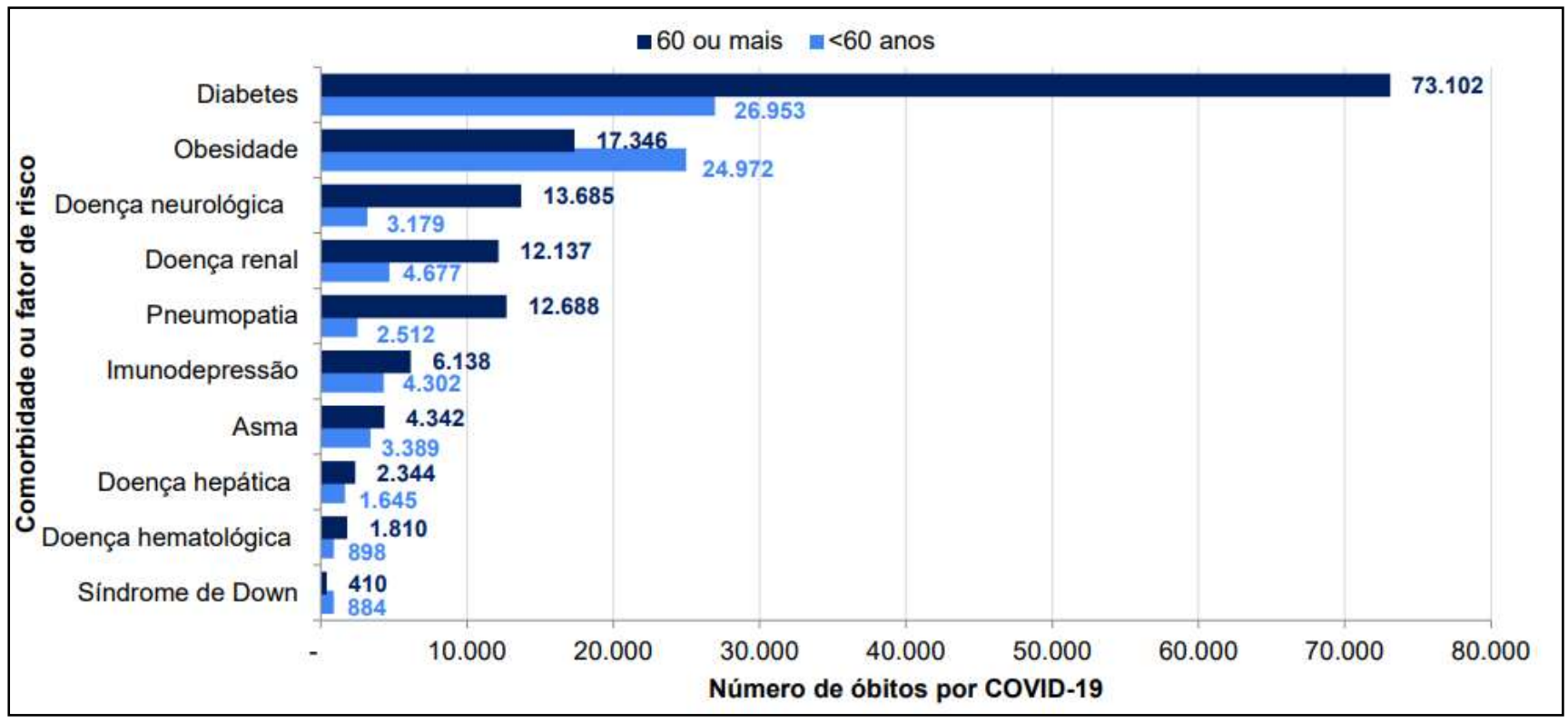

Fonte: Sistema de Informação de Vigilância da Gripe (SIVEP-Gripe, 2021). Dados atualizados em 30 de agosto de 2021.

Diante desses dados analisados, é possível concluir que a obesidade é um fator de risco relevante, presente na mortalidade por infecção do SARS-CoV-2 e suas complicações. É importante relatar que desde o primeiro óbito visto nos Boletins Epidemiológicos (03 de abril de 2020) até o SE-34 houve uma alteração na incidência da COVID-19, segundo sexo, idade e região. Logo, desde o primeiro caso notificado até o dia 28 de agosto de 2021, é notório que a região Centro-oeste apresentou maior incidência de adultos obesos (21,02\%) em comparação com as demais regiões, assim como, a maior taxa de mortalidade por COVID-19 (3,6 óbitos/100 mil hab.). A incidência de obesos em relação ao sexo é a mesma, porém, a incidência de óbitos ainda é maior no sexo masculino (186.004 casos). Por fim, com relação a idade o percentual de obesos foi entre 55 a 64 anos (42,3\%), seguido por 45 a 54 anos (24,5\%), e o número de óbitos na obesidade foi maior dentre os menores de 60 anos, chegando a 24.972 casos (Brasil - Ministério da Saúde, 2021b).

\section{Conclusão}

Observou-se com os dados analisados que grande parte da população está obesa (20,3\%), ou com excesso de peso $(55,4 \%)$ além das comorbidades associadas, e apresentam maior chance de morte por COVID-19. Ao avaliar os fatores epidemiológicos da COVID-19 é fundamental atentar para a caracterização da contaminação com intuito de uma intervenção e atenção à saúde, para reduzir o número de óbitos. No entanto, as ações de enfrentamento a COVID-19 só serão efetivas se houver um comprometimento da tríade governo-sociedade-setor privado, seguindo os protocolos e orientações da Organização Mundial da Saúde. Novos estudos deverão analisar mais dados atualizados para verificar se a tendência observada no presente estudo permanece, para que novas condutas possam ser tomadas pela equipe multiprofissional que trabalha arduamente no combate a COVID-19.

\section{Referências}

ABESO - Associação Brasileira para o Estudo da Obesidade e da Síndrome Metabólica. (2021). Mapa da obesidade. https://abeso.org.br/obesidade-e-sindromemetabolica/mapa-da-obesidade/ 
Research, Society and Development, v. 11, n. 1, e10911123432, 2022

(CC BY 4.0) | ISSN 2525-3409 | DOI: http://dx.doi.org/10.33448/rsd-v11i1.23432

Bolsoni-Lopes, A., Furieri, L. B. \& Alonso-Vale, M. I. C. (2021). Obesidade e a covid-19: uma reflexão sobre a relação entre as pandemias. Revista Gaúcha de Enfermagem, 42

Brasil - Ministério da Saúde. (2020a). Boletim epidemiológico especial, número 06, 3 de abril de 2020. Doença pelo Coronavírus COVID-19. https://portalarquivos.saude.gov.br/images/pdf/2020/April/03/BE6-Boletim-Especial-do-COE.pdf.

Brasil - Ministério da Saúde. (2020b). Secretaria de Vigilância em Saúde, Boletim Epidemiológico Volume 51, No 27 Jul. 2020. https://antigo.saude.gov.br/images/pdf/2020/July/09/Boletim-epidemiologico-SVS-27-06.07.2020.pdf.

Brasil - Ministério da Saúde. (2021a). Boletim epidemiológico especial, número 52. Doença pelo Coronavírus COVID-19. Semana Epidemiológica 8 (21 a 27/2/2021). https://www.gov.br/saude/pt-br/media/pdf/2021/marco/05/boletim_epidemiologico_covid_52_final2.pdf.

Brasil - Ministério da Saúde. (2021b). Boletim epidemiológico especial, número 28. Doença pelo Coronavírus COVID-19. Semana Epidemiológica 34 (16 a 22/08)). https://antigo.saude.gov.br/images/pdf/2020/August/27/Boletim-epidemiologico-COVID-28-FINAL-COE.pdf.

Cândido, J. A. B., Moreira, M. R. C., Alexandre, S. F., Parente, N. C., \& Cavalcante, N. R. P. (2021). Obesidade em paciente com prognóstico de gravidade para Covid-19. Research, Society and Development, 10(10), e459101019121. https://doi.org/10.33448/rsd-v10i10.19121

Chait, A., \& den Hartigh, L. J. (2020). Adipose Tissue Distribution, Inflammation and Its Metabolic Consequences, Including Diabetes and Cardiovascular Disease. Frontiers in cardiovascular medicine, 7, 22. https://doi.org/10.3389/fcvm.2020.00022

Domingues, C. M. A. S. (2021). Desafios para a realização da campanha de vacinação contra a COVID-19 no Brasil.

Ferreira, A. P. D. S., Szwarcwald, C. L. \& Damacena, G. N. (2019). Prevalência de obesidade e fatores associados na população brasileira: um estudo com dados da Pesquisa Nacional de Saúde 2013. Revista Brasileira de Epidemiologia, 22.

Fonseca-Alaniz, M. H., Takada, J., Alonso-Vale, M. I. C. \& Lima, F. B. (2006). O tecido adiposo como centro regulador do metabolismo. Arquivos Brasileiros de Endocrinologia \& Metabologia, 50, 216-229.

Gregório, M. J., Santos, A., \& Graça, P. (2021). Obesity and COVID-19: Present and Future. Acta Médica Portuguesa, 34(5), 329-331.

Guan, W. J., Ni, Z. Y., Hu, Y., Liang, W. H., Ou, C. Q., He, J. X., ... \& Zhong, N. S. (2020). Clinical characteristics of coronavirus disease 2019 in China. New England journal of medicine, 382(18), 1708-1720.

Halpern, B., Louzada, M. L. D. C., Aschner, P., Gerchman, F., Brajkovich, I., Faria-Neto, J. R., ... \& Franco, O. H. (2021). Obesidade e COVID-19 na América Latina: a tragédia de duas pandemias - Documento oficial da Federação Latino-Americana de Sociedades de Obesidade. Comentários sobre obesidade, 22 (3), e13165.

Lana, R. M., Coelho, F. C., Gomes, M. F. D. C., Cruz, O. G., Bastos, L. S., Villela, D. A. M., \& Codeço, C. T. (2020). Emergência do novo coronavírus (SARSCoV-2) e o papel de uma vigilância nacional em saúde oportuna e efetiva. Cadernos de Saúde Pública, 36, e00019620.

Malavazos, A. E., Romanelli, M. M. C., Bandera, F., \& Iacobellis, G. (2020). Visando o tecido adiposo em COVID - 19. Obesidade (Silver Spring, MD). Malta, D. C., Szwarcwald, C. L., Barros, M. B. D. A., Gomes, C. S., Machado, Í. E., Souza, P. R. B. D., ... \& Gracie, R. (2020). The COVID-19 Pandemic and changes in adult Brazilian lifestyles: a cross-sectional study, 2020. Epidemiologia e Serviços de Saúde, 29.

SIVEP-Gripe - Sistema de Informação de Vigilância da Gripe. (2021). https://sivepgripe.saude.gov.br/sivepgripe/

Souza, L. E. P. F. D. \& Buss, P. M. (2021). Desafios globais para o acesso equitativo à vacinação contra a COVID-19. Cadernos de Saúde Pública, 37, e00056521.

VIGITEL - Vigilância de Fatores de Risco e Proteção para Doenças Crônicas por Inquérito Telefônico. (2019). https://bvsms.saude.gov.br/bvs/publicacoes/vigitel_brasil_2019_vigilancia_fatores_risco.pdf

WHO - World Health Organization. (2020). Obesity and overweight. https://www.who.int/newsroom/fact-sheets/detail/obesity-and-overweight 